\title{
Energy Minimization with One Dot Fuzzy Initialization for Marine Oil Spill Segmentation
}

\author{
Peng Ren, SeniorMember, IEEE, Min Xu, Yunhua Yu, Fang Chen, Xiangyuan Jiang and Erfu Yang
}

\begin{abstract}
Detecting marine oil spill regions in synthetic aperture radar (SAR) images has always been posed as a segmentation problem in terms of minimizing a certain energy function(al). As most energy minimization problems do not have analytical solutions, minimizing an energy function(al) is usually achieved in an iterative numerical manner. In this scenario, one key factor that affects the segmentation accuracy is the initialization for starting or constraining the numerical iterations. To guarantee accurate segmentation, a proper initialization that characterizes the marine oil spill layouts in a SAR image is required. However, marine oil spill regions are always complicatedly shaped, and it is inefficient to manually devise precise initializations for capturing various marine oil spill shapes. In order to address this problem and render efficient and robust segmentation, we develop a one dot fuzzy initialization strategy. In contrast to the normal practice of manually labeling a large amount of pixels (possibly lines or cycles of pixels subject to strict spatial conditions) as initialization, our strategy just requires one arbitrary pixel within a marine oil spill region as the initial dot. The intuition of our strategy is that the fuzzy connectedness between an arbitrary initial dot and the rest pixels enables the derivation of a physically homogeneous region which is consistent for initializing the energy minimization. In the light of this observation, we develop schemes for exploiting the one dot derived region to initialize both level sets for minimizing continuous energy functionals and graph cuts for minimizing discrete energy functions. Experimental results validate the robustness of our one dot fuzzy initialization strategy.
\end{abstract}

Index Terms-Marine oil spill segmentation, energy minimization, one dot fuzzy initialization, fuzzy connectedness.

\section{INTRODUCTION}

In recent decades, marine oil spill accidents have frequently occurred at different scales and have caused various damages to the natural environment [1]. Satellite-based synthetic aperture radar (SAR) has the advantage of all-weather and all-time operation and plays an important role in observing environment and targets [2][3][4]. SAR also provides an important

This work was supported in part by the National Natural Science Foundation of China (Grant No.: 61671481), the Royal Society of Edinburgh and the National Natural Science Foundation of China joint project (Grant No.: 6161101383), the Fundamental Research Funds for the Central Universities (Project No. 18CX05014A) and Qingdao Applied Fundamental Research (Grant No.: 16-5-1-11-jch).

Peng Ren, Min Xu, Fang Chen and Xiangyuan Jiang are with the College of Information and Control Engineering, China University of Petroleum (East China), Qingdao 266580, China (email: pengren@upc.edu.cn; nyxumin@126.com; chenfangupc@163.com; jxy@upc.edu.cn).

Yunhua $\mathrm{Yu}$ is with the College of Information and Control Engineering, China University of Petroleum (East China), Qingdao 266580, China, and also with the Shengli College, China University of Petroleum (East China), Dongying 257097, China (email: yuyunhua@upc.edu.cn).

Erfu Yang is with the Department of Design, Manufacture and Engineering Management, University of Strathclyde, Glasgow G1 1XJ, UK (email: erfu.yang@strath.ac.uk). means for monitoring marine oil spills [5][6]. It is vital to accurately observe the oil spills through SAR images in a timely manner for the purpose of both damage assessment and spread control. Therefore, developing intelligent algorithms for segmenting marine oil spill regions from SAR images has been an important research topic in the literature of ocean remote sensing.

In the research literature, the majority of oil spill study based on SAR data lies in the investigation of the physic characteristics of oil spills for reflecting different types of electromagnetic waves. One major physical feature for oil spill analysis based on SAR data is the non-Bragg scattering phenomenon caused by oil spills. The capillary and short gravity waves on the ocean surface give rise to Bragg scattering that is sensed by SAR. On the other hand, marine oil spills damp out the Bragg scattering, resulting in dark patches in SAR images. The non-Bragg scattering regions provide indications for observing oil spills based on SARimages. Recently, more sophisticated oil and electromagnetic wave relationships have been explored, and especially the polarimetric characteristics of oil spills have been comprehensively investigated. In this regard, Nunziata et al. [7], Buono et al. [8], Bandiera et al. [9][10], Minchew et al. [11], Collins et al. [12], Brekke et al. [13] and Espeseth et al. [14] have conducted studies that represent state of the art oil spill observation research based on polarimetric SAR data. The polarimetry based strategies enhance oil spill observations in images through multiple polarimetric channel analysis such that basic image processing techniques such as thresholding [15] and $K$-means clustering [8] are easily applied to detecting oil spills in the enhanced representations.

On the other hand, researchers mainly from the image processing and machine learning community have been working on developing more sophisticated oil spill segmentation methods for accurately detecting oil spill regions in SAR images. In this scenario, oil spill segmentation is formulated in terms of energy minimization, in which an energy function(al) measures the segmentation characteristics such as fitness and similarity with respect to the oil spills. The energy function(al)s can be roughly classified into continuous functionals and discrete functions, depending on whether the variables are continuous or discrete. The two types of energies are formulated in different forms and require different numerical computation schemes. Especially, they model images in different perspectives and have their own advantages separately. Continuous energy minimization characterizes the topological similarity between the segmentation and image representation and tends to result in detailed oil spill region contours. Discrete 
energy minimization takes advantage of fast approximation strategies and renders efficient computation for segmentation.

As continuous and discrete energy minimization methods have their unique advantages, both of them have been studied in the oil spill segmentation literature. Mdakane et al. [16] incorporated a region-based signed pressure force function into the level set continuous energy functional for detecting oil slicks from moving vessels. Chen et al. [17] described how to generalize level set continuous energy miminization strategies to segment oil spill regions from blury images. Ren et al. [18] proposed to smooth both image and cost volume for discrete energy functions, and apply the graph cut algorithm for obtaining optimal segmentation. In contrast to state-of-the-art deep learning strategies (e.g. [19]) that require labelled oil spill images for training a complicated model, the energy minimization methods directly conduct segmentation on single SAR observation according to the oil spill intrinsic characteristics. The availability of oil spill images is not as easy as that of everyday images taken by ordinary cameras, and training a segmentation model based on a big amount (segmented) oil spill images seems an inappropriate mission. An energy minimization method turns out to be a more suitable strategy for segmenting oil spill regions in SAR images, because it straightforwardly operates on one image without the training procedure.

However, it is observed that the accuracy of energy minimization segmentation heavily relies on the prior knowledge provided for initializing the energy minimization [20]. In practice, a considerable number of pixels (possibly lines or cycles of pixels subject to spatial conditions) are manually labeled as initialization. For continuous energy minimization, level set methods are widely used for numerical computation where level set evolution is commonly initialized by a regular contour such as a rectangle box (partially) surrounding an object. For discrete energy minimization, graph cut methods are widely used for approximation and the initialization requires labeling parts of foreground and background pixels separately for constraining graph cut computation. Though an energy function(al) initialized by similar initializations can result in almost identical results for normal object segmentation, slightly different initializations may lead to totally different segmented oil spill regions. This is because unlike regularly formed normal objects, one oil spill region usually manifests itself in an irregular shape with an arbitrary curved contour. For example, it has been observed that regular initializations such as rectangle boxes are not sufficient to characterize the sophisticatedly shaped oil spills [20]. For instance, Fig. 5(a) illustrates one marine oil spill SAR image and its manually segmented oil spill regions. Energy minimization methods require initializations to follow the shape of an oil spill region for obtaining accurate segmentation. However, manually devising precise initializations for guiding segmentation is inefficient for practical use. In contrast, one common practice is to manually develop the initial contours or initial labels through coarse human observation of oil spill regions. The initializations thus obtained tend to exhibit various forms and are not reliable for achieving accurate segmentation. Therefore, the requirement for both the efficiency of devising an initialization and its reliability for guiding the accurate segmentation appears to be a paradox. On the other hand, there are a number of initialization-free solution proposed for energy minimization (especially for the continuous energy minimization methods such as level sets). One pioneering study in the literature is the active contour without edges [21], which avoids manual initialization by a gradient-free strategy. Recently, the Otsu's method, which employs certain thresholding scheme for detecting initial contours, has been broadly employed for automatically initializing level sets for oil spill segmentation [16][22]. Though these methods efficiently avoid manual initializations, their practical implementations do not always result in acceptable segmentation results, especially in segmenting irregular shapes such as oil spills.

In order to achieve both efficient and robust initialization for accurate segmentation, we propose a one dot fuzzy initialization scheme. Our method just requires an arbitrary pixel within one marine oil spill region as prior knowledge, which turns out an efficient scheme. Furthermore, it results in a consistent region which is reliable for accurate segmentation. Key to the effectiveness of our strategy is that the fuzzy connectedness between an arbitrary initial dot and the rest pixels enables the derivation of a physically homogeneous region. We exploit the physically homogeneous region as a consistent initial region for establishing initializations for both continuous and discrete energy minimization strategies. Specifically, we use such initialization to drive both the level set and graph cut computations and achieve consistent and accurate segmentation results for detecting oil spill regions in SAR images.

\section{ENERGy MiNIMIZATION}

We describe the continuous and discrete energy minimization strategies separately in this section. Specifically, we describe the constructions of region scalable fitting (RSF) continuous energy functional and the pairwise graph based discrete energy function, and then explain how to render energy minimization via level sets and graph cuts, respectively. Finally, we give a comparison between the level set and graph cut image segmentation schemes.

\section{A. Continuous Energy Minimization}

In this subsection, we describe the RSF level set method [23] for continuous energy minimization. The construction of the RSF energy functional and the minimization of the energy functional via level sets are presented separately in the image segmentation scenario.

1) Region Scalable Fitting Energy Functional: For an image $I$ with its pixel intensity at $(x, y)$ denoted as $I(x, y)$, one level set method employs a level set function $\phi(x, y)$ as a segmentation indicator. Geometrically, $\phi$ manifests itself as a surface in a three-dimensional space. In numerical computation, $\phi$ can be thought of a matrix with the same size as the image $I$. For an optimal level set function, $\phi(x, y) \geq 0$ indicates that the pixel $I(x, y)$ is segmented into the marine oil spill region, otherwise it is segmented into background. The curve obtained in terms of the intersection between $\phi$ 
and the zero plane (i.e. $\phi(x, y)=0$ ) is referred to as the zero level set of $\phi(x, y)$, and it indicates the contour of an oil spill region. RSF is an approach to establishing an energy functional (i.e. objective function) with respect to $\phi$. The RSF energy functional is defined as follows:

$$
\begin{aligned}
E_{\epsilon}\left(\phi, q_{1}, q_{2}\right)= & \sum_{i=1}^{2} \lambda_{i} \iint\left(\iint \omega_{i} M_{i}^{\epsilon}(\phi(u, v)) \mathrm{d} u \mathrm{~d} v\right) \mathrm{d} x \mathrm{~d} y \\
& +\nu \iint\left|\nabla H_{\epsilon}(\phi(x, y))\right| \mathrm{d} x \mathrm{~d} y
\end{aligned}
$$

where

$$
\omega_{i}=K_{\sigma}(x-u, y-v)\left|I(u, v)-q_{i}(x, y)\right|^{2}
$$

Here $K_{\sigma}$ is a nonnegative kernel function and $\sigma$ is its scale parameter. Additionally, $M_{1}^{\epsilon}(\phi)=H_{\epsilon}(\phi), M_{2}^{\epsilon}(\phi)=1-$ $H_{\epsilon}(\phi) . H_{\epsilon}(\phi)$ is a smooth Heaviside function where $\epsilon$ is a parameter for smoothing the step change.

$$
H_{\epsilon}(\phi)=\frac{1}{2}\left[1+\frac{2}{\pi} \arctan \left(\frac{\phi}{\epsilon}\right)\right]
$$

The derivative of $H_{\epsilon}(\phi)$ is:

$$
\delta_{\epsilon}(\phi)=\frac{\partial H_{\epsilon}}{\partial \phi}=\frac{1}{\pi} \frac{\epsilon}{\epsilon^{2}+\phi^{2}} .
$$

The integrations in (1) operate over the spatial domain of the whole image. $M_{i}^{\epsilon}$ controls the integration domain for $w_{i}$ such that the integration with respect to $(u, v)$ just takes place within the $\phi \geq 0$ image region if $i=1$ or within the $\phi<0$ image region if $i=2$. $w_{i}$ measures the fitness between an image approximation quantity $q_{i}(x, y)$ and the original image $I$ within the $\phi \geq 0$ region if $i=1$ or within the $\phi<0$ region if $i=2$. It is in such a manner that the level set $\phi$ characterizes the region scalable fitness between the segmentation and the image.

To preserve the regularity of the level set function and maintain a stable level set evolution, it is necessary for the energy functional to involve a level set regularization term $R(\phi)$ as follows:

$$
R(\phi)=\iint \frac{1}{2}(|\nabla \phi(x, y)-1|)^{2} \mathrm{~d} x \mathrm{~d} y .
$$

Here more comprehensive regularization schemes such as the double-well potential [24] can be employed. For simplicity and without loss of generality, we keep using the distance regularization (5) in our work. Specifically, we aim to propose a consistent and efficient initialization strategy for starting the level set evolution, and the distance regularization is capable of avoiding the degraded representation and the reinitialization inefficiency during the level set evolution. Therefore, our proposed strategy and the distance regularization complements each other in constructing an overall level set segmentation framework.

The overall RSF energy functional given by:

$$
E_{C}\left(\phi, q_{1}, q_{2}\right)=E_{\epsilon}\left(\phi, q_{1}, q_{2}\right)+\eta R(\phi)
$$

where $\eta$ is a positive balancing parameter. The energy functional (6) is classified as continuous energy because its variables are intrinsically continuous.
2) Level Set Evolution for Continuous Energy Minimization: The basic idea of the level set method is to formulate the contour motion as the evolution of a level set function for the purpose of minimizing the energy functional (6). The functions $q_{1}$ and $q_{2}$ that minimize the energy functional $E_{C}\left(\phi, q_{1}, q_{2}\right)$ for a fixed $\phi$ satisfy the following Euler-Lagrange equations:

$$
\iint K_{\sigma}(x-u, y-v) M_{i}(\phi(u, v))\left[I(u, v)-q_{i}(x, y)\right] \mathrm{d} u \mathrm{~d} v=0
$$

and $q_{i}(x, y)$ is obtained as follows:

$$
q_{i}(x, y)=\frac{K_{\sigma}(x, y) *\left[M_{i}(\phi(x, y)) I(x, y)\right]}{K_{\sigma}(x, y) * M_{i}(\phi(x, y))}, i=1,2 .
$$

The functions $q_{1}$ and $q_{2}$ are the weighted averages of the pixel intensities in a neighborhood of $(x, y)$, and the size of $q_{i}$ is proportional to the scale parameter $\sigma$.

Keeping $q_{1}$ and $q_{2}$ fixed, we use the standard gradient decent method to minimize the energy functional $E_{C}\left(\phi, q_{1}, q_{2}\right)$ with respect to $\phi$, and the required partial derivative is:

$$
\begin{aligned}
\frac{\partial E_{C}}{\partial \phi}= & \delta_{\epsilon}(\phi)\left(\lambda_{1} e_{1}-\lambda_{2} e_{2}\right)-\nu \delta_{\epsilon}(\phi) \operatorname{div}\left(\frac{\nabla \phi}{|\nabla \phi|}\right) \\
& -\eta\left[\nabla^{2} \phi-\operatorname{div}\left(\frac{\nabla \phi}{|\nabla \phi|}\right)\right]
\end{aligned}
$$

where $e_{1}$ and $e_{2}$ are the functions as follows:

$$
e_{i}(x, y)=\iint \omega_{i} \mathrm{~d} u \mathrm{~d} v, i=1,2 .
$$

The gradient descent update for $\phi$ is:

$$
\phi_{t}(x, y)=\phi_{t-1}(x, y)-\left.\alpha \frac{\partial E_{C}}{\partial \phi}\right|_{t-1}
$$

where $\alpha$ is the learning rate. The convergence of the level set evolution according to (8) and (11) achieves the minimization of the RSF energy functional (6). Detailed explanations about the RSF level sets are referred to [23]. The zero level set of the converged level set function yields the contour for the marine oil spill region in one SAR image.

The initial level set function for starting the evolution (11) plays an important role in accurate segmentation. One widely accepted initial level set construction strategy is to manually establish a rectangle box that (partially) surrounds a marine oil spill region. The manually initialized level set is supposed to capture the oil spill shapes as much as possible. However, manual initializations are somewhat subjective and arbitrary such that appropriateness of the manually initialized level sets cannot be guaranteed. The evolving iterations in (11) starting from different initial level sets are likely to converge to different final level sets, thus resulting in different oil spill segmentation results. One reason for the segmentation variation is the approximation applied in the numerical computation. The continuous operators such as divergence are all approximated by numerical operations in the evolution such that one small initial difference may lead to considerable segmentation difference via a series of numerical approximations. This heavily hinders the level set method from practical use. The contribution of our work overcomes this inefficiency by developing one efficient and reliable initialization method based on just one dot, which will be presented in Section III. 


\section{B. Discrete Energy Minimization}

In this subsection, we describe the graph cut method [25] for discrete energy minimization. The construction of a discrete energy function and the minimization of the energy function via graph cuts are presented separately in the image segmentation scenario.

1) Pairwise Energy Function: For an image $I$, let $N$ be the set of pixels and $\mathcal{N}$ be the set of all pairs of neighboring pixels. The image segmentation is performed by assigning each pixel $i \in N$ a label $l_{i} \in L$ with binary value 0 or 1 , which indicates the pixel belonging to "oil spill regions" or "background". The label set $L$ represents the segmentation.

To measure the disagreement between segmentation and image at the individual pixel level, a unary potential term is defined as follows:

$$
\mathcal{E}_{i}\left(l_{i}\right)=l_{i}\left(\vartheta-I_{i}\right)+\left(1-x_{i}\right)\left(I_{i}-\vartheta\right)
$$

where $\vartheta$ is an empirical threshold and $I_{i}$ is the intensity value at the pixel $i$. The unary potential favors segmentation subject to thresholding each individual pixel by $\vartheta$. If the discrete energy function consists of just the unary potentials, the segmentation is reduced to basic thresholding segmentation, which tends to be susceptible to noise. To address this ineffectiveness, the discrete energy function normally involves pairwise potentials as follows:

$$
\begin{aligned}
\mathcal{E}_{i, j}\left(l_{i}, l_{j}\right)= & {\left[l_{i}\left(1-l_{j}\right)+\left(1-l_{i}\right) \cdot l_{j}\right] . } \\
& \exp \left[-\frac{\left(I_{i}-I_{j}\right)^{2}}{\sigma^{2}}\right] \cdot \frac{1}{d_{i, j}}
\end{aligned}
$$

where $d_{i, j}=\sqrt{\left(x_{i}-x_{j}\right)^{2}+\left(y_{i}-y_{j}\right)^{2}}$ represents the distance between the two pixels, $\sigma$ is a scaling factor, and $(i, j)$ is a pair of neighboring pixels included in $\mathcal{N}$. The pairwise potential favors identical labels for neighboring pixels by assigning zero penalty. On the other hand, it assigns a pair of distinct labels a penalty which follows the intensity dissimilarity and spatial distance between two pixels.

The overall discrete energy function is as follows:

$$
E_{D}\left(l_{1}, l_{2}, \cdots, l_{N}\right)=\sum_{i=1}^{N} \mathcal{E}_{i}\left(l_{i}\right)+\gamma \sum_{\{i, j\} \subset \mathcal{N}} \mathcal{E}_{i, j}\left(l_{i}, l_{j}\right)
$$

where $\gamma$ is a positive balaning parameter. The energy (14) is referred to pairwise energy function, because each individual potential term takes no more than two label variables. As the variables in (14) are all discrete with the value 0 or 1 , it is classified into the category of discrete energy. The pairwise energy function can be thought of a composition of unary potential terms for penalizing individual disagreements and pairwise potential terms for smoothing. The unary potentials and pairwise potentials coplay with each other in the overall energy function (14). The unary potentials tend to assign the oil spill label to every pixel with intensity greater than a threshold and may inevitably encourage segmenting noise as oil spills. As noisy pixels tend to exhibit considerable difference from their neighboring pixels, the pairwise potentials avoid the noisy effect of unary potentials by favoring neighboring pixels with identical labels. On the other hand, pairwise potentials tend to assign identical labels to all pairs of pixels. Unary potentials balance the over-smoothing effect of pairwise potentials by penalizing the disagreement between segmentation and image intensity for each individual pixel. In this way, pixels on both sides of an oil boundary are encouraged to be labeled differently.

Recently, potential terms with orders higher than pairwise [18][26][27] have been studied for establishing more complicated energy functions. However, these higher order energy functions are transformed into pairwise energy functions in the forms similar to (14) for the purpose of practical optimization. Therefore, for simplicity and without loss of generality, we use the pairwise energy function (14) in our work for developing a new initialization scheme.

2) Graph Cuts for Discrete Energy Minimization: Once a discrete energy function (14) has been established for an image $I$, the graph cut algorithm is used for minimizing (14) to obtain optimal segmentation. To this end, a graph associated with the image $I$ is established. The graph has $N+2$ vertices with $N$ of them representing $N$ pixels and the rest two representing two terminals $O$ and $B$. The terminals $O$ and $B$ represent the binary segmentation labels oil (i.e. $l=0$ ) and background (i.e. $l=1$ ), respectively. Each terminal vertex connects to every pixel vertex through an edge. For an edge connecting the terminal vertex $O$ and the pixel vertex $i$, the edge weight is $\mathcal{E}_{i}(0)$. Similarly, for an edge connecting the terminal vertex $B$ and the pixel vertex $i$, the edge weight is $\mathcal{E}_{i}(1)$.

The $N$ pixel vertices interconnect with one another by edges in the graph. The edge weight between the pixel vertices $i$ and $j$ are the pairwise potential with identical labels $\mathcal{E}_{i, j}(0,0)$ or $\mathcal{E}_{i, j}(1,1)$. Note that $\mathcal{E}_{i, j}(0,0)$ or $\mathcal{E}_{i, j}(1,1)$ have the same value according to (13).

For a graph characterized by the discrete energy function (14), the graph cut algorithm is applied and the min-cut, which disconnects the terminal vertices $O$ and $B$ by cutting off subset of edges with the minimum cost of edge weights, is obtained. The pixel vertices still connecting to the terminal vertex $O$ is segmented into the oil spill region and the pixel vertices still connecting to the terminal vertex $B$ is segmented into the background. The graph cut optimization is implemented in terms of the max-flow/min-cut algorithm which involves a large load of iterations such as spanning search trees. Furthermore, to render efficient computation in image segmentation, the reusage of search trees for approximation is exploited to avoid expensive computational overheads [28]. To guide the energy minimization process for achieving max-flow/min-cut that properly follows the image characteristics, certain initial labels are required as hard labeling constraints. Specifically, before applying the graph cut algorithm, certain pixels in the oil region and in the background are manually labeled as 0 and 1, respectively. Specifically, in the graph, one pixel vertex manually labeled as oil has an extremely big weight edge to the terminal vertex $O$ and has a zero weight edge to the terminal vertex $B$. Therefore, the edge between the pixel vertex and terminal vertex $O$ can never be cut off in the graph cut algorithm. Similarly, the pixel vertex manually labeled as background has an extremely big weight edge to the terminal vertex $B$ and has a zero weight edge to the terminal 
vertex $O$. Therefore, the edge between the pixel vertex and terminal vertex $B$ can never be cut off in the graph cut algorithm. Finally, the iterative operations for max-flow/mincut just operate under the constraint of the initial labels.

Fig. 1 illustrates an example of segmenting a nine pixel image based on graph cuts with initial labels. Fig. 1(a) indicates the initial labels for segmentation. The white line indicates that the two pixels on the top left are manually labeled as oil. The dash line right-angle indicates that the three pixels in the bottom right corner are manually labeled as background. Constrained by the initial labels, the graph is constructed as illustrated in Fig. 1(b). The thickness of each edge reflects its weight between each pixel and the labeled terminals. Zero weight edges are not drawn. Fig. 1(c) indicates the min-cut obtained according to Fig. 1(b). Fig. 1(d) is the final segmentation. The shaded area represents the segmented objective and the blank space represents the background.

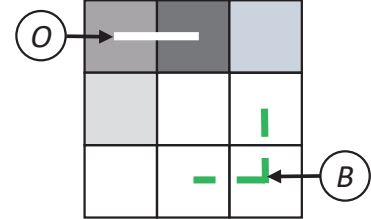

(a) Image with initial labels

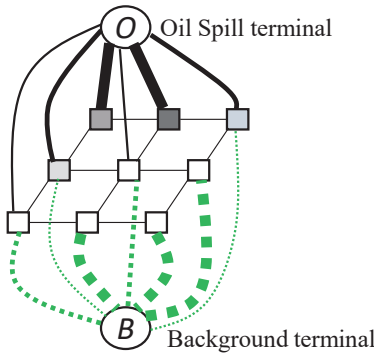

(c) Graph construction

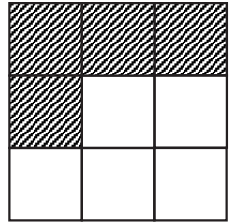

(b) Segmented results

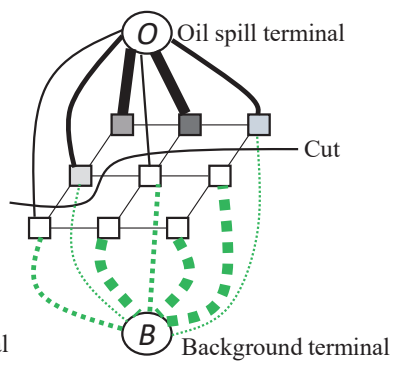

(d) Graph cut
Fig. 1: An example for graph cuts.

The manually initialized labels are supposed to provide sufficient indication for separating the oil spill and background. However, manual labels based on human coarse observation cannot thoroughly consider the discriminative characteristics between oil and background, and may not always provide sufficient indication for constraining the segmentation. Different sets of manual initial labels tend to result in different graph cut segmentation results and some of them are highly inaccurate. We will describe in Section III how to develop an efficient one dot initialization scheme which provides consistent and sufficient initial oil labels for graph cuts.

\section{Comparison between Level Sets and Graph Cuts}

Level sets perform energy minimization via numerically solving variational functional equations. On the other hand, graph cuts take advantage of discrete operations and render more efficient computation. Level sets use comprehensively shaped surface for characterizing curves in images and are generally endowed with more representative power than graph cuts in depicting irregular regions [29].
The initialization for level sets is to provide a level set function for starting the iterative evolution. The initialization for graph cuts is to manually label certain pixels as oil and background separately, which constrain the optimization process. Therefore, the initializations for level sets and graph cuts are intrinsically different. However, in practice, both initializations are implemented via marking image pixels. We will show that our new strategy provides a consistent and robust method for initializing both level sets and graph cuts for marine oil spill segmentation.

\section{ONE DOT FUZZY INITIALIZATION}

This section describes a novel one dot fuzzy initialization strategy. We first explain how to develop an initial region based on one dot fuzzy connectedness. We then describe how to initialize level sets and graph cuts based on the initial region separately. Finally, we discuss the reason for the consistency of the fuzzy initialization.

\section{A. One Dot Fuzzy Connectedness for Initial Region}

Though both the level sets and graph cuts are reasonably formulated and can achieve state of the art segmentation performance in various image processing tasks, their accuracy highly relies on the initialization, especially for segmenting irregular shapes such as marine oil spill regions. Different initializations may result in tremendously different marine oil spill segmentation results. To guarantee accurate segmentation, it is expected that the initialization consistently follows the marine oil spill spatial layouts. In the light of this observation, we propose a one dot fuzzy initialization strategy which exploits the fuzzy connectedness [30] between pixels and is capable of establishing consistent initial regions via different initial dots within one marine oil region.

We commence by computing the fuzzy adjacency $\rho_{i, j}$ between the pixels at $\left(x_{i}, y_{i}\right)$ and $\left(x_{j}, y_{j}\right)$ as follows:

$$
\rho_{i, j}=\left\{\begin{array}{c}
\frac{1}{1+\beta_{1} d_{i, j}}, d_{i, j} \leq 1 \\
0, \text { otherwise. }
\end{array}\right.
$$

where $\beta_{1}$ is a nonnegative constant.

Then the fuzzy affinity $a_{i, j}$ between the pixels at $\left(x_{i}, y_{i}\right)$ and $\left(x_{j}, y_{j}\right)$ is defined as:

$$
a_{i, j}=\frac{\rho_{i, j}}{1+\beta_{2}\left|I\left(x_{i}, y_{i}\right)-I\left(x_{j}, y_{j}\right)\right|}
$$

where $\beta_{2}$ is a nonnegative constant

Suppose that the one initial dot used as the prior knowledge within the oil spill region is at $\left(x_{0}, y_{0}\right)$ in the SAR image. We compute the fuzzy connectedness $\mu_{\left(x_{0}, y_{0}\right)}(x, y)$ of one pixel at $(x, y)$ with respect to the selected initial dot at $\left(x_{0}, y_{0}\right)$ as follows:

$$
\mu_{\left(x_{0}, y_{0}\right)}(x, y)=\max _{p \in P}\left(\min _{(i, j) \in p} a_{i, j}\right)
$$

where $P$ denotes the set of all possible paths between the pixel at $\left(x_{0}, y_{0}\right)$ and the pixel at $(x, y)$, and $p$ denotes an individual path between the pixel at $\left(x_{0}, y_{0}\right)$ and the pixel at $(x, y)$. Here, a path is a sequence of spatially neighboring pixels, and in (17) $a_{i, j}$ denotes the fuzzy affinity of a pair of neighboring pixels 
at $\left(x_{i}, y_{i}\right)$ and $\left(x_{j}, y_{j}\right)$ along the path $p$. The strength of a path is the smallest affinity of pairwise pixels along the path. The operation min in (17) computes the strength of a path $p$. The operation max in (17) selects the strongest path for characterizing the fuzzy connectedness.

The concept of fuzzy connectedness is derived from the theory of fuzzy sets. In the fuzzy set formulation, $\left\{\left(x_{0}, y_{0}\right),(x, y)\right\}$ is an element in a fuzzy set, and the fuzzy connectedness $\mu_{\left(x_{0}, y_{0}\right)}(x, y)$ is its membership function characterizing the 2-ary fuzzy relation. The theory of fuzzy sets elaborates many characteristics of fuzzy connectedness such as physical homogeneity, which plays an important role in our initialization scheme.

The computation of (17) is implemented via dynamic programming, which has the optimal substructure property that a subpath of a strongest path is itself a strongest path.

Suppose $\left(x_{1}, y_{1}\right)$ is one spatially neighboring pixel to the initial dot $\left(x_{0}, y_{0}\right)$. The fuzzy connectedness of $\left(x_{1}, y_{1}\right)$ with respect to $\left(x_{0}, y_{0}\right)$ is:

$$
\mu_{\left(x_{0}, y_{0}\right)}\left(x_{1}, y_{1}\right)=a_{0,1}
$$

which forms one starting point for the following computations.

Suppose the pixel at $\left(x_{i}, y_{i}\right)$ is one whose fuzzy connectedness with respect to the initial dot has not been computed yet in the dynamic programming but whose neighboring pixels' connectednesses with respect to the initial dot have already been computed. Let $\mathcal{N}_{i}$ denote the set of spatially neighboring pixels of the pixel at $\left(x_{i}, y_{i}\right)$. Let $i_{\mathcal{N}}$ be one element in $\mathcal{N}_{i}$, i.e. $i_{\mathcal{N}} \in \mathcal{N}_{i}$. The problem of computing the fuzzy connectedness (17) is broken down into the following two simpler subproblems as follows:

$$
\begin{gathered}
i_{\mathcal{N}}^{*}=\underset{i_{\mathcal{N}} \in \mathcal{N}_{i}}{\arg \max }\left\{a_{i_{\mathcal{N}}, i}\right\} \\
\mu_{\left(x_{0}, y_{0}\right)}\left(x_{i}, y_{i}\right)=\min \left\{\mu_{\left(x_{0}, y_{0}\right)}\left(x_{i_{\mathcal{N}}^{*}}, y_{i_{\mathcal{N}}^{*}}\right), a_{i_{\mathcal{N}}^{*}, i}\right\}
\end{gathered}
$$

Solving the subproblems (19) and (20) in a recursive manner results in the dynamic programming solution to (17).

\section{B. One Dot Fuzzy Initial Contour for Level Sets}

For an oil spill SAR image and an initial dot at $\left(x_{0}, y_{0}\right)$ within the oil spill region, we define the initial level set function $\phi_{0}(x, y)$ with $t=0$ as follows:

$$
\phi_{0}(x, y)= \begin{cases}+2, & \mu_{\left(x_{0}, y_{0}\right)}(x, y) \geq \theta \\ -2, & \mu_{\left(x_{0}, y_{0}\right)}(x, y)<\theta\end{cases}
$$

where $\theta$ is an empirical thresholding parameter. The zero level set of $\phi_{0}(x, y)$ is the initial contour.

We use the initial contour thus obtained to start the level set evolution. The convergence of evolution yields the final level set which indicates oil spill regions in terms of the domain with the level set value greater than zero. One exemplary flowchart for marine oil spill segmentation based on level sets with one dot fuzzy initialization is illustrated in Fig. 2. The white dot indicates the initial dot. The yellow region is the initial region derived from the initial dot according to fuzzy connectedness. The white line indicates the initial contour for level set evolution.

\section{One Dot Fuzzy Region for Graph Cuts}

For an oil spill SAR image with an initial dot at $\left(x_{0}, y_{0}\right)$ in the oil spill region, we computed the fuzzy connectedness $\mu_{\left(x_{0}, y_{0}\right)}(x, y)$. We then assign the initial label $l=0$ (i.e. oil spill) to the pixels satisfying $\mu_{\left(x_{0}, y_{0}\right)}(x, y) \geq \theta$. We refer to the region of pixels obtained by the thresholding $\mu_{\left(x_{0}, y_{0}\right)}(x, y) \geq \theta$ as the fuzzy region. The fuzzy region, which is labeled as oil spill, behaves as the initially labelled oil spill region for constraining graph cuts.

We use comparatively arbitrary lines of pixels as initial labels for the background because the fuzzy region plays a more dominating role in constraining the graph cut algorithm. Based on these initial labels, we operate the graph cut algorithm on the image and obtain the final segmentation. One exemplary flowchart for marine oil spill segmentation based on graph cuts with one dot fuzzy initialization is illustrated in Fig. 3. The red region is the fuzzy region with respect to the one initial dot. It is used as the oil spill initial label for graph cuts. The green dash line is the background initial label which is determined in a comparatively arbitrary manner.

\section{Observations}

Both level sets and graph cuts exploit the region $\mu_{\left(x_{0}, y_{0}\right)}(x, y) \geq \theta$ for initialization, and we refer to it as the initial region. One advantage of the initial region is that it is itself consistent and is not susceptible to the location of the initial dot within the oil spill region. Specifically, differently located initial dots derive identical initial regions in terms of fuzzy connectedness. The reason for the consistency of initial regions is that fuzzy connectedness comprehensively characterizes the physical homogeneities of an image. The fuzzy connectedness characterizes the global relations between pixels in terms of feature similarity and spatial adjacency. The initial contour derived via the fuzzy connectedness with respect to one initial dot thus follows the global spatial layout of the marine oil spill and is insusceptible to the variation of initial dot location within the oil spill region. Detailed explanations and theoretical proofs for the physical homogeneities of fuzzy connectedness can be found in [31]. Therefore, when we use one dot fuzzy initialization in practice, we do not need to consider too much about the exact location of the initial dot. As long as it is located within the oil spill region, it can derive identical initial regions which form consistent initializations for energy minimization. This advantage facilitates energy minimization methods for practical oil spill segmentation.

The term one dot fuzzy initialization implies two underlying meanings. First, the initial region is derived from the initial dot in terms of fuzzy connectedness. Second and more important, the initial dot does not require a precisely arranged location. Specifically, as long as it is within the oil spill region, its exact location can be a bit arbitrary, which reflects certain 'fuzziness'.

\section{EXPERIMENTAL EVALUATIONS}

To validate the effectiveness of the proposed initialization scheme, we test our strategy on SAR images containing differently shaped marine oil spill regions. We use the SAR images 


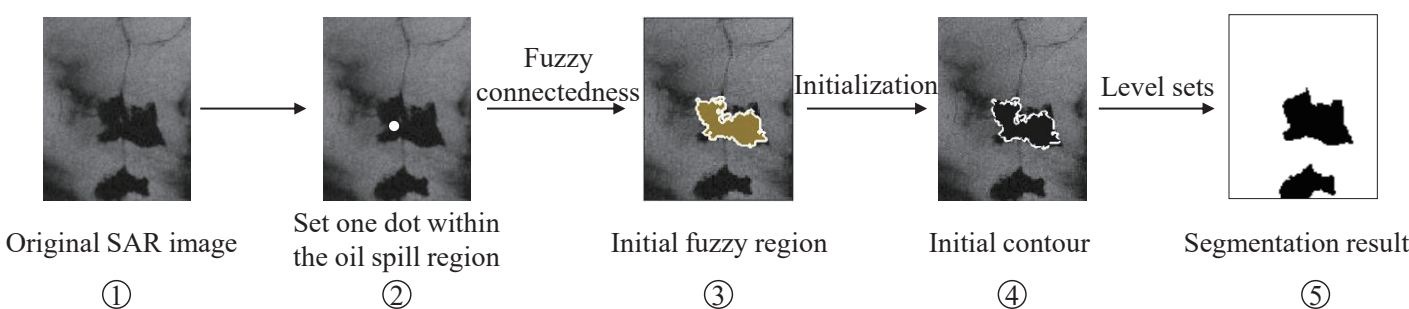

Fig. 2: One dot initialization example for continuous energy minimization.

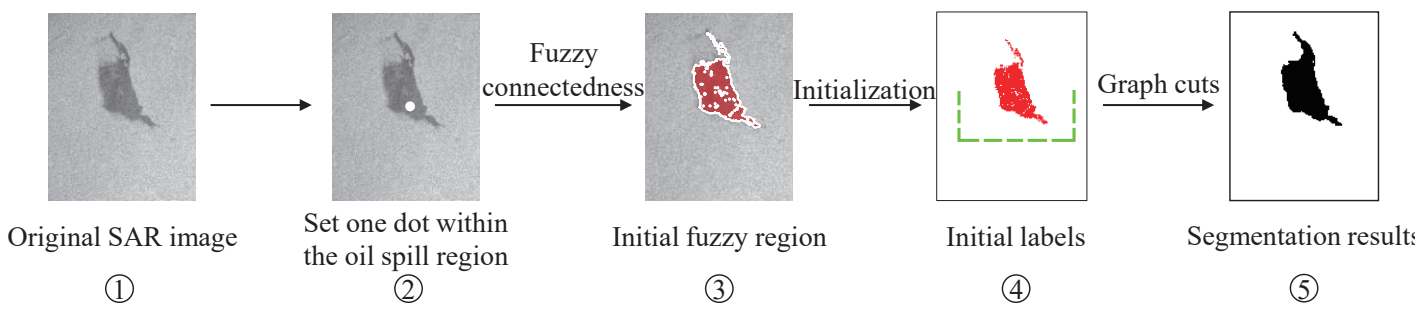

Fig. 3: One dot initialization example for discrete energy minimization.

with VV polarization obtained from the Northwest Pacific Action Plan (NOWPAP) database in our experiments. The images are C-band SAR images from the ERS-1(European Remote Sensing Satellite-1) and ERS-2(European Remote Sensing Satellite-2) satellites. The experiments are implemented by using Matlab 2016b with an embedded C++ compiler.

NOWPAP was adopted in September 1994 as a part of the Regional Seas Program of the United Nations Environment Program (UNEP). The implementation of NOWPAP contributes to the Global Program of Action (GPA) for the protection of the marine environment from land-based activities in the northwest Pacific region. The geographical scope of NOWPAP covers the marine environment and coastal zones from about 121 degree $\mathrm{E}$ to 143 degree $\mathrm{E}$ longitude, and from approximately 33 degree $\mathrm{N}$ to 52 degree $\mathrm{N}$ latitude.

In order examine the performance of state of the art methods and make empirical comparison between our method and them, we commence by testing two existing initializationfree methods [21][16] on the SAR data. These methods automatically determine the initializations without prior manual labeling and are thus considered to be initialization free. Fig. 4 illustrates the oil spill segmentation results based on the two initialization-free methods. The first row illustrates the segmentation results based on the initialization-free method presented in [21]. The second row illustrates the RSF level set segmentation results based on the automatic initialization presented in [16]. Furthermore, to make quantitative experimental evaluation, we compute the recall ( $\frac{\# \text { correctly segmented pixels }}{\# \text { oil spill pixels }}$ ) and precision ( $\frac{\# \text { correctly segmented pixels }}{\# \text { segmented pixels }}$ ) for segmentation results of alternative comparison methods. The quantitative experimental evaluations on the segmentation accuracies of the two initialization-free methods are given in TABLE I. We observe from Fig. 4 that the initialization-free methods tend to induce the over-segmentation of oil spills. They segment more areas rather than true oil spills into the oil class. The observation is also validated by the quantitative evaluation in TABLE I, where both methods result in high recalls but low precisions. Such contrastive results in terms of recall and precision reflect that the initialization-free methods have limited capability of guiding accurate oil spill segmentation.

We use the region scalable fitness level set [23] and the higher order graph cut [26] as baselines for validating the effectiveness of our method, because they are popular energy minimization methods with state of the art performance and also with publicly available code. As our method focuses on the initialization and does not intervene in the energy minimization inference procedures, other alternative level set or graph cut methods can also be tested indiscriminately as baselines.

Fig. 5 illustrates the segmentation results based on alternative strategies. To validate the effectiveness of our initialization scheme for improving level set segmentation accuracy, we conduct the initializations by using two slightly different rectangle boxes (illustrated in Figs. 5(b) and (c)) and two different arbitrary dots within one marine oil spill region (illustrated in Figs. 5(d) and (e)). The two slightly different initial contours result in the segmentations with considerable differences. The one dot fuzzy initialization scheme overcomes this deficiency because the initial regions derived from the two different initial dots are quite identical, resulting in consistent segmented oil regions. Furthermore, the segmentation results in Figs. 5(d) and (e) derived from one dot fuzzy initialization are much more accurate than those in Figs. 5(b) and (c) derived from the initial box contours.

To validate the effectiveness of our initialization scheme for improving graph cut segmentation accuracy, we conduct the oil label initializations by using two different small red regions (illustrated in Figs. 5(g) and (h)) and two different arbitrary dots within one marine oil spill region (illustrated in Figs. 5(i) and (j)). To make fair comparisons, we use the same green dash lines as background label initialization for the four cases. The two different red regions result in the segmentations with considerable differences and great inaccuracy. The one dot fuzzy initialization scheme overcomes this deficiency because 
the two sets of initial oil labels derived from the two different initial dots separately are quite identical, resulting in consistent and accurate segmented oil regions in the end.

Figs. 6, 7, 8, 9, 10 and 11 illustrate the experimental results in the same manner with Figs. 5. All these visual results qualitatively validate the effectiveness of our initialization scheme over the manual initializations. TABLE II and TABLE III provide the quantitative experimental results in terms of recall and precision.

Compared with the segmentation results of the initializationfree methods in Fig. 4 and TABLE I, our method is more effective. Specifically, different from the high contrast between recall and precision based on the initialization-free methods, our method achieves balance between the two metrics with high accuracies. Therefore, our method is more robust and accurate than the two initialization-free methods in segmenting oil spills. Furthermore, it is interesting to observe from TABLEs II and III that all the one dot fuzzy initializations overwhelmingly outperform the manual initializations in terms of recall, except those in Fig. 8 for level sets and those in Fig. 6 for graph cuts. In these cases, though manual initializations achieve recalls comparable with the one dot fuzzy initializations, their precisions are fairly lower. On the other hand, some manual initializations (especially for graph cuts) achieve slightly better precisions than the one dot fuzzy initializations. However, these tiny precision superiorities are traded off by the extreme low recalls. The high precision results from severe undersegmentation. For example, the segmentation results based on manually initialized graph cuts in Figs. 8 (g) and (h) have high precisions because their segmented regions just cover small parts of the true oil spill region. However, they do not segment large parts of the true oil spill region and their recalls are thus extremely low. Therefore, the overall performance of manual initialization is inferior to that of the one dot initialization in terms of both robustness and consistency.

It can be seen from both qualitative and quantitative experimental evaluation that our one dot fuzzy initialization has greatly improved the robustness and accuracy of both the continuous and discrete energy minimization methods for marine oil segmentation.

\section{Conclusions}

In contrast to the existing energy minimization schemes which require a considerable amount of prior knowledge about oil spills and background, the one dot fuzzy initialization just uses one dot as prior knowledge. Therefore, the proposed scheme does not require an number of pixels manually labeled for initializing energy minimization. It thus facilitates energy minimization methods for practical marine oil spill segmentation. Furthermore, the one dot fuzzy initialization exploits the fuzzy connectedness between pixels and yields a consistent initial region regardless of the dot location within the oil spill region. Therefore, the segmentation based on our one dot fuzzy initialization is more robust than those with subjective manual initializations. We have described how to use the proposed one dot fuzzy initialization to initialize both continuous level set energy minimization and discrete graph cut energy minimization. The experimental results have validated that both level sets and graph cuts yield robust and accurate marine oil spill segmentation based on the one dot fuzzy initialization.

SAR polarimetric features have the potential to discriminate dark oil spill lookalike areas from true oil spill regions in SAR images. In our future work, we will extend our method to processing SAR polarimetric feature maps and develop more effective oil spill detection schemes.

\section{REFERENCES}

[1] P. F. Kingston, "Long-term environmental impact of oil spills," Spill Science \& Technology Bulletin, vol. 7, no. 1, pp. 53-61, 2002.

[2] D. Velotto, C. Bentes, B. Tings, and S. Lehner, "First comparison of Sentinel-1 and TerraSAR-X data in the framework of maritime targets detection: South Italy case," IEEE Journal of Oceanic Engineering, vol. 41, no. 4, pp. 993-1006, 2016.

[3] H. Li, J. Wu, W. Perrie, and Y. He, "Wind speed retrieval from hybridpol compact polarization synthetic aperture radar images," IEEE Journal of Oceanic Engineering, to appear in 2017.

[4] T. Soukissian, F. Karathanasi, and P. Axaopoulos, "Satellite-based offshore wind resource assessment in the Mediterranean Sea," IEEE Journal of Oceanic Engineering, vol. 42, no. 1, pp. 73-86, 2017.

[5] B. Fiscella, A. Giancaspro, F. Nirchio, P. Pavese, and P. Trivero, "Oil spill detection using marine SAR images," International Journal of Remote Sensing, vol. 21, no. 18, pp. 3561-3566, 2000.

[6] A. H. Solberg, C. Brekke, and P. O. Husoy, "Oil spill detection in Radarsat and Envisat SAR images," IEEE Transactions on Geoscience and Remote Sensing, vol. 45, no. 3, pp. 746-755, 2007.

[7] F. Nunziata, M. Migliaccio, and X. Li, "Sea oil slick observation using hybrid-polarity SAR architecture," IEEE Journal of Oceanic Engineering, vol. 40, no. 2, pp. 426-440, 2015.

[8] A. Buono, F. Nunziata, M. Migliaccio, and X. Li, "Polarimetric analysis of compact-polarimetry SAR architectures for sea oil slick observation," IEEE Transactions on Geoscience and Remote Sensing, vol. 54, no. 10, pp. 5862-5874, 2016.

[9] F. Bandiera and G. Ricci, "Slicks detection on the sea surface based upon polarimetric SAR data," IEEE Geoscience and Remote Sensing Letters, vol. 2, no. 3, pp. 342-346, 2005.

[10] F. Bandiera, A. Masciullo, and G. Ricci, "A bayesian approach to oil slicks edge detection based on SAR data," IEEE Transactions on Geoscience and Remote Sensing, vol. 52, no. 5, pp. 2901-2909, 2014.

[11] B. Minchew, C. E. Jones, and B. Holt, "Polarimetric analysis of backscatter from the Deepwater Horizon oil spill using L-band synthetic aperture radar," IEEE Transactions on Geoscience and Remote Sensing, vol. 50, no. 10, pp. 3812-3830, 2012.

[12] M. J. Collins, M. Denbina, B. Minchew, C. E. Jones, and B. Holt, "On the use of simulated airborne compact polarimetric SAR for characterizing oil-water mixing of the Deepwater horizon oil spill," IEEE Journal of Selected Topics in Applied Earth Observations and Remote Sensing, vol. 8, no. 3, pp. 1062-1077, 2015.

[13] C. Brekke, C. E. Jones, S. Skrunes, B. Holt, M. Espeseth, and T. Eltoft, "Cross-correlation between polarization channels in SAR imagery over oceanographic features," IEEE Geoscience and Remote Sensing Letters, vol. 13, no. 7, pp. 997-1001, 2016.

[14] M. M. Espeseth, S. Skrunes, C. E. Jones, C. Brekke, B. Holt, and A. P. Doulgeris, "Analysis of evolving oil spills in full-polarimetric and hybrid-polarity SAR," IEEE Transactions on Geoscience and Remote Sensing, vol. 55, no. 7, pp. 4190-4210, 2017.

[15] A. Lupidi, D. Staglianò, M. Martorella, and F. Berizzi, "Fast detection of oil spills and ships using SAR images," Remote Sensing, vol. 9, no. 3 , pp. 230-247, 2017.

[16] L. W. Mdakane and W. Kleynhans, "An image-segmentation-based framework to detect oil slicks from moving vessels in the southern african oceans using SAR imagery," IEEE Journal of Selected Topics in Applied Earth Observations \& Remote Sensing, vol. 10, no. 6, pp. 2810-2818, 2017.

[17] F. Chen, H. Zhou, C. Grecos, and P. Ren, "Segmenting oil spills from blurry images based on alternating direction method of multipliers," IEEE Journal of Selected Topics in Applied Earth Observations and Remote Sensing, doi:10.1109/JSTARS.2018.2833485, 2018. 
[18] P. Ren, M. Di, H. Song, C. Luo, and C. Grecos, "Dual smoothing for marine oil spill segmentation," IEEE Geoscience and Remote Sensing Letters, vol. 13, no. 1, pp. 82-86, 2016.

[19] X. Yu, H. Zhang, C. Luo, H. Qi, and P. Ren, "Oil spill segmentation via adversarial $f$-divergence learning," IEEE Transactions on Geoscience and Remote Sensing, doi:10.1109/TGRS.2018.2803038, 2018.

[20] M. Xu, Y. Yu, F. Chen, X. Jiang, P. Ren, and E. Yang, "Level sets with one dot fuzzy initialization for marine oil spill segmentation," in Procedings of the IEEE/MTS OCEANS 2017-Aberdeen Conference, 2017, pp. 1-4.

[21] T. F. Chan and L. A. Vese, "Active contours without edges," IEEE Transactions on Image Processing, vol. 10, no. 2, pp. 266-277, 2001.

[22] N. Otsu, "A threshold selection method from gray-level histograms," IEEE Transactions on Systems Man \& Cybernetics, vol. 9, no. 1, pp. 62-66, 2007.

[23] C. Li, C.-Y. Kao, J. C. Gore, and Z. Ding, "Minimization of regionscalable fitting energy for image segmentation," IEEE transactions on image processing, vol. 17, no. 10, pp. 1940-1949, 2008.

[24] C. Li, C. Xu, C. Gui, and M. D. Fox, "Distance regularized level set evolution and its application to image segmentation," IEEE transactions on image processing, vol. 19, no. 12, pp. 3243-3254, 2010.

[25] Y. Boykov and G. Funka-Lea, "Graph cuts and efficient ND image segmentation," International journal of computer vision, vol. 70, no. 2, pp. 109-131, 2006.

[26] P. Kohli, A. Osokin, and S. Jegelka, "A principled deep random field model for image segmentation," in Proceedings of the IEEE Conference on Computer Vision and Pattern Recognition, 2013, pp. 1971-1978.

[27] Y. Kitamura, Y. Li, W. Ito, and H. Ishikawa, "Data-dependent higherorder clique selection for artery-vein segmentation by energy minimization," International Journal of Computer Vision, vol. 117, no. 2, pp. 142-158, 2016.

[28] Y. Boykov and V. Kolmogorov, "An experimental comparison of mincut/max-flow algorithms for energy minimization in vision," IEEE transactions on pattern analysis and machine intelligence, vol. 26, no. 9, pp. 1124-1137, 2004

[29] Y. Mei and X. Wang, "A level set method for structural topology optimization and its applications," Advances in Engineering Software, vol. 35 , no. 7, pp. 415-441, 2004.

[30] J. K. Udupa and S. Samarasekera, "Fuzzy connectedness and object definition: theory, algorithms, and applications in image segmentation," Graphical models and image processing, vol. 58, no. 3, pp. 246-261, 1996.

[31] J. K. Udupa and P. K. Saha, "Fuzzy connectedness and image segmentation," Proceedings of the IEEE, vol. 91, no. 10, pp. 1649-1669, 2003. 

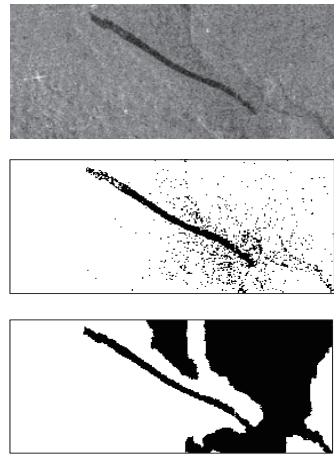
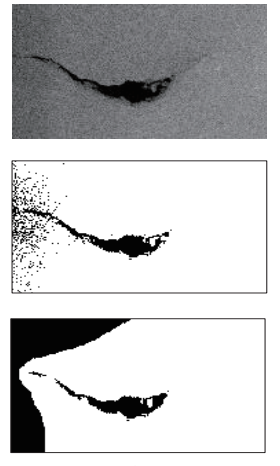

(b)
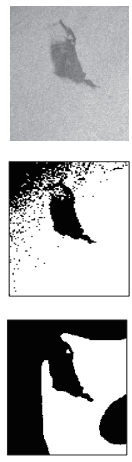

(c)
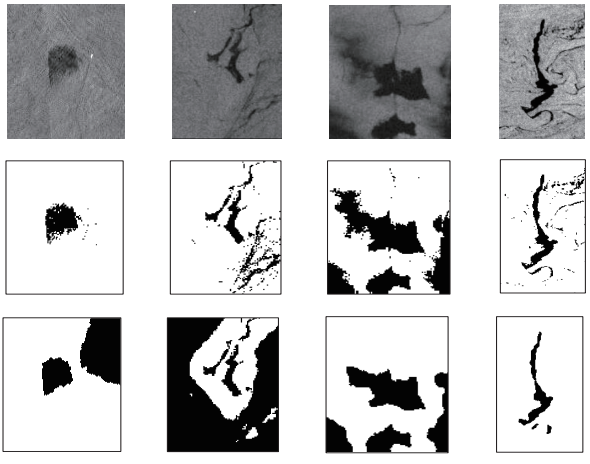

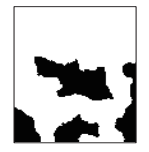

(f)

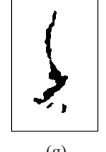

(g)

Fig. 4: Marine oil spill segmentation results the initialization-free methods. First row: Segmentations based on Chan et al. [21]. Second row: Segmentations based on Mdakane et al. [16].

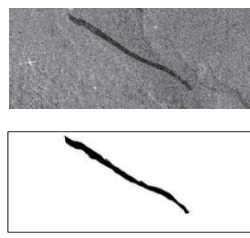

(a)
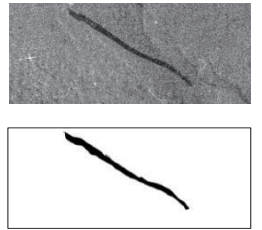

(f)
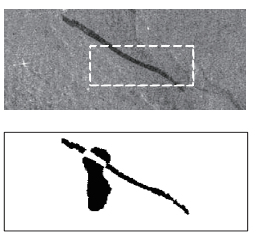

(b)
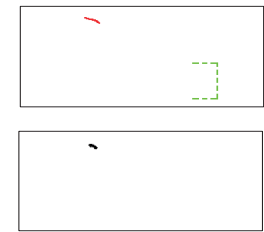

(g)
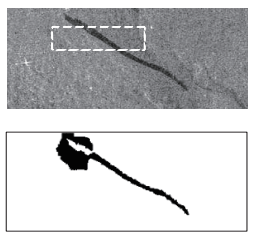

(c)

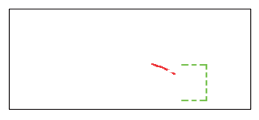

-
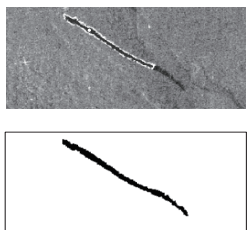

(d)
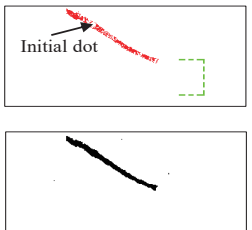

(i)
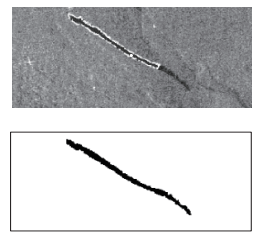

(e)
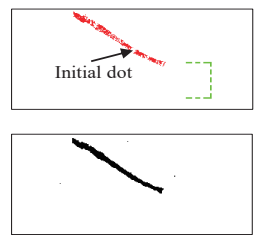

(j)

Fig. 5: Marine oil spill segmentation results using level sets and graph cuts separately: (a) and (f) illustrate an original SAR image and its ground truth segmentation; (b) and (c) illustrate two different initial level set functions (i.e. initial contours displayed as white boxes overlaid on the SAR images) and their segmented oil spill regions based on level set evolutions; (d) and (e) illustrate two initial dots (displayed as white solid dots within the oil spill regions) along with their derived initial contours (displayed as white dash curves overlaid on the SAR images), and their segmented oil spill regions based on level set evolutions; (g) and (h) illustrate two different sets of initial labels (i.e. red regions for oil and green dash lines for background) and their segmented oil spill regions based on graph cuts; (i) and (j) illustrate two initial dots (displayed as white solid dots) along with their derived initial oil labels (displayed as red regions), and their segmented oil spill regions based on graph cuts.
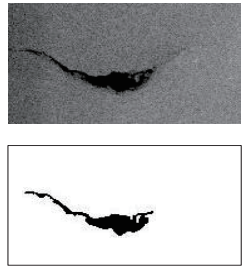

(a)
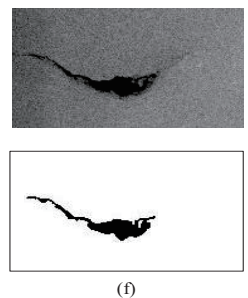
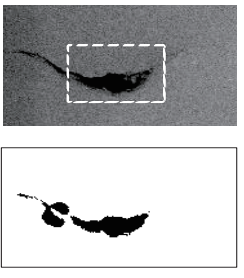

(b)
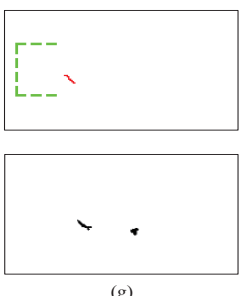
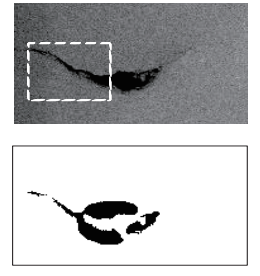

(c)
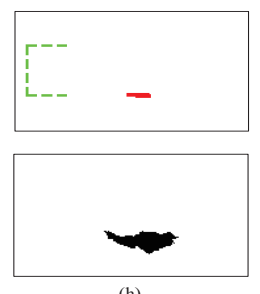

(h)
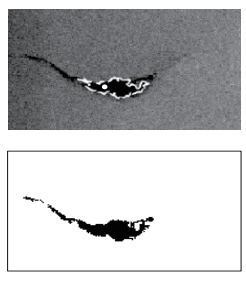

(d)
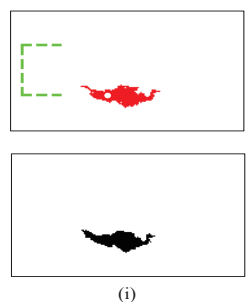
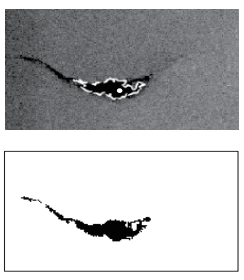

(e)
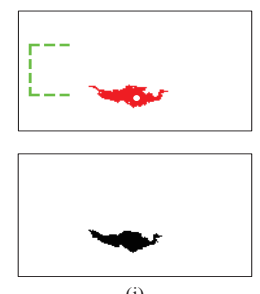

Fig. 6: Marine oil spill segmentation results using level sets and graph cuts separately. Subfigures are indexed and entitle in the same ways with Fig. 5 . 


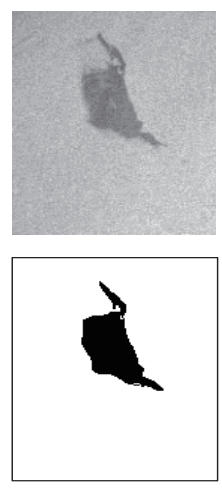

(a)
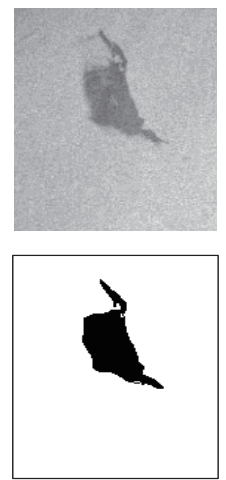

(f)
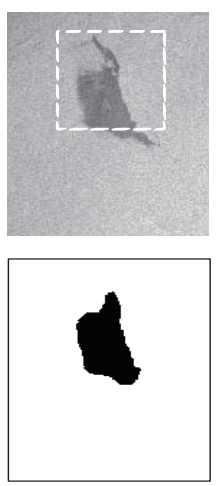

(b)
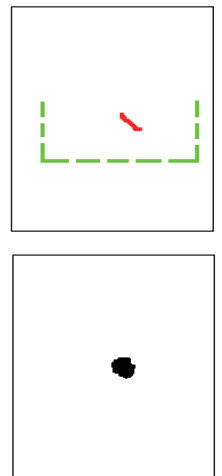

(g)
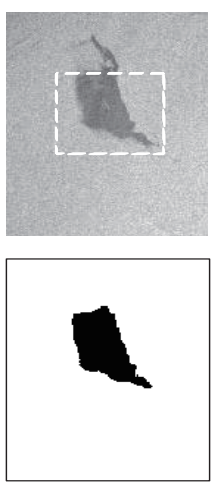

(c)
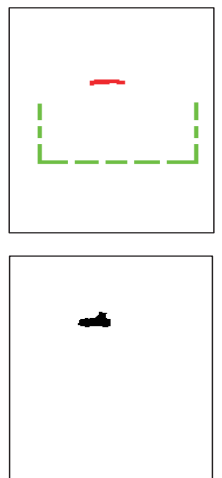

(h)
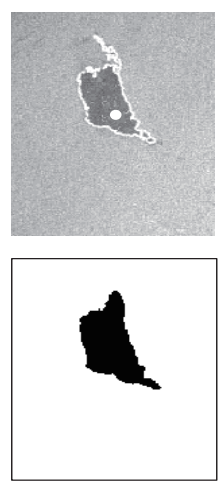

(d)
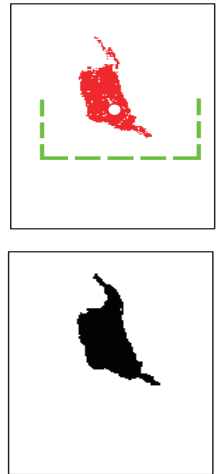

(i)
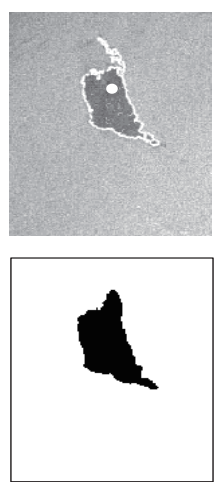

(e)
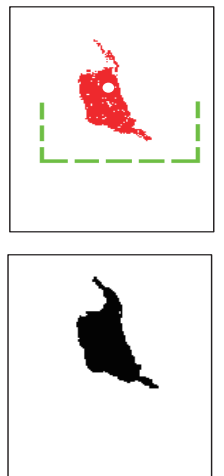

(j)

Fig. 7: Marine oil spill segmentation results using level sets and graph cuts separately. Subfigures are indexed and entitle in the same ways with Fig. 5.
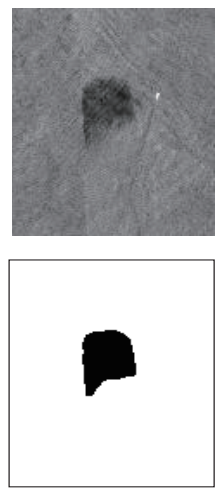

(a)
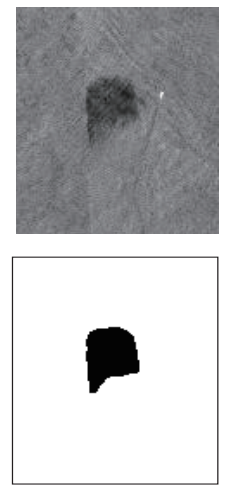

(f)
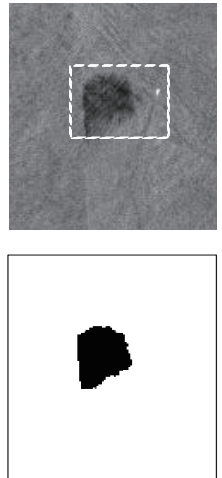

(b)
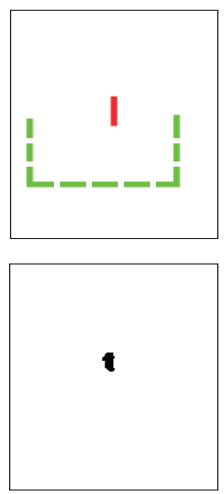

(g)
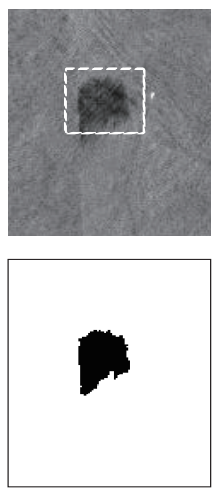

(c)
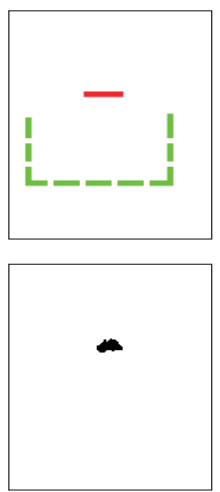

(h)
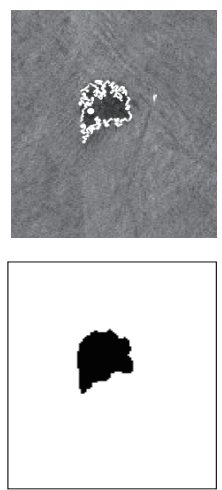

(d)
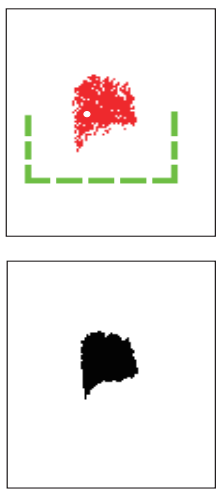

(i)
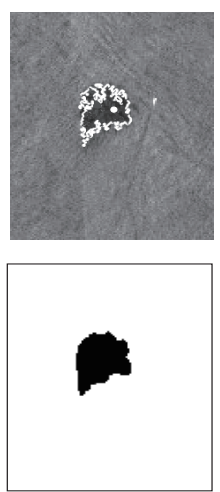

(e)
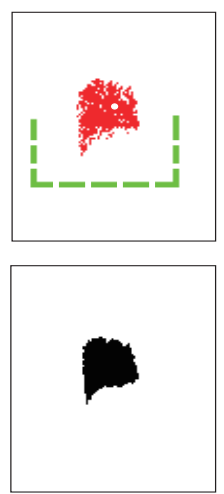

(j)

Fig. 8: Marine oil spill segmentation results using level sets and graph cuts separately. Subfigures are indexed and entitle in the same ways with Fig. 5 . 

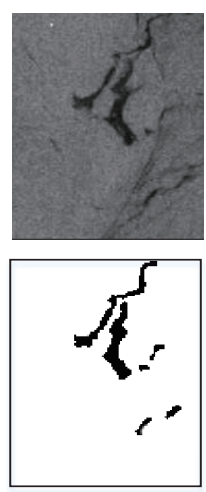

(a)
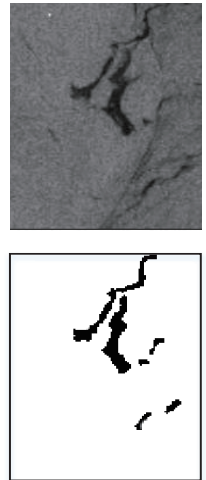

(f)
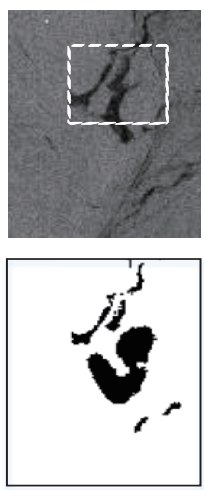

(b)
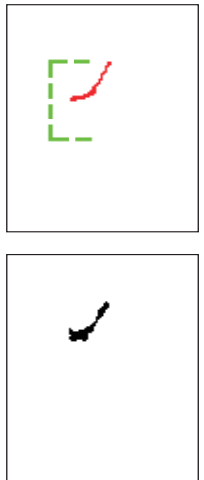

(g)
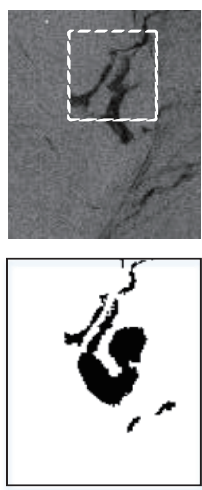

(c)
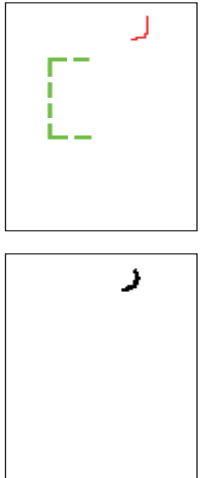

(h)
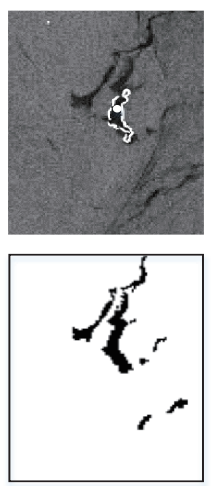

(d)
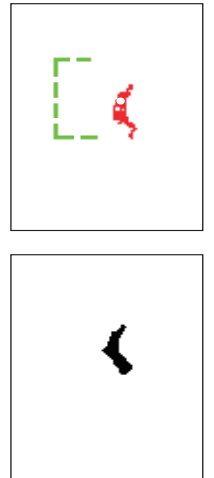

(i)
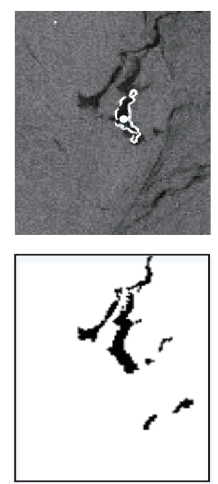

(e)
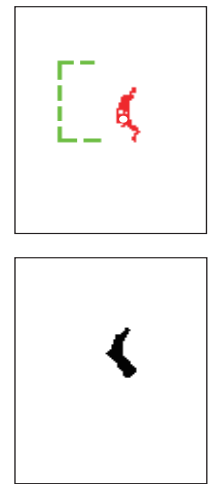

(j)

Fig. 9: Marine oil spill segmentation results using level sets and graph cuts separately. Subfigures are indexed and entitle in the same ways with Fig. 5.
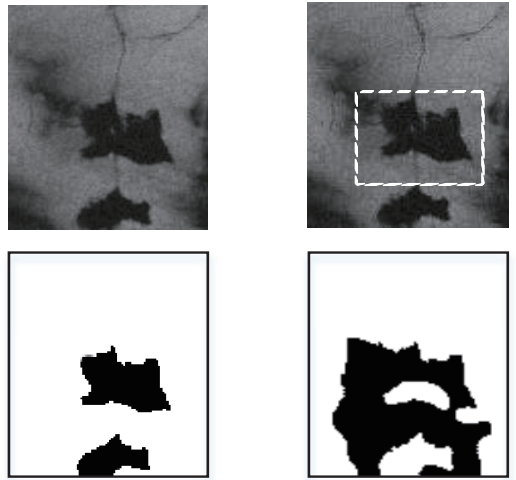

(a)
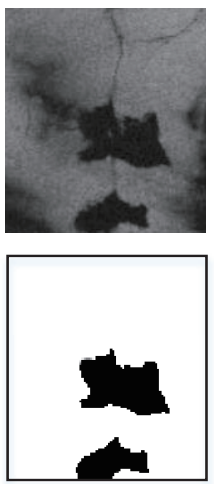

(f)

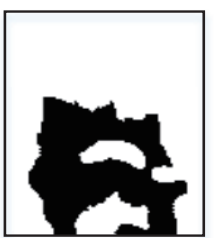

(b)
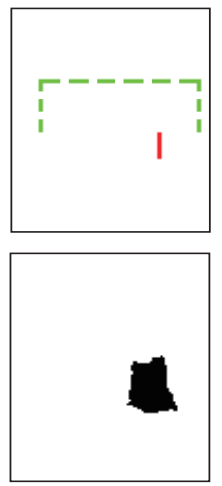

(g)
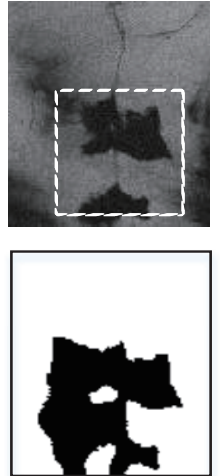

(c)
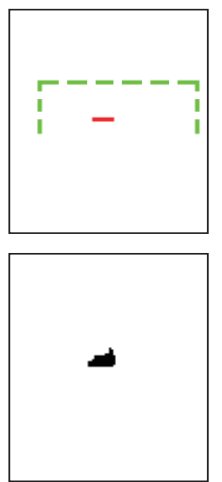

(h)
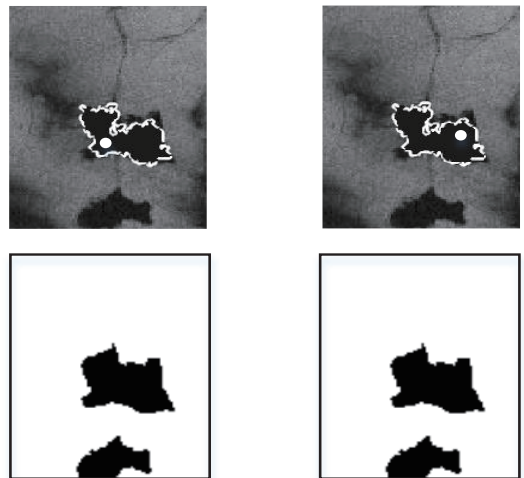

(d)
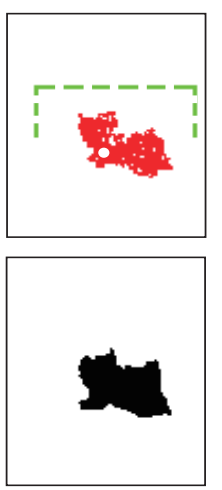

(i)

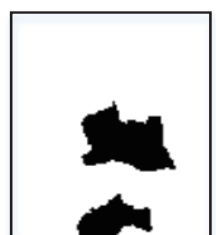

(e)
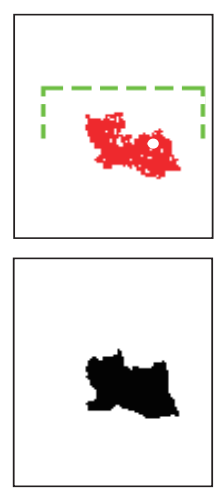

(j)

Fig. 10: Marine oil spill segmentation results using level sets and graph cuts separately. Subfigures are indexed and entitle in the same ways with Fig. 5 . 

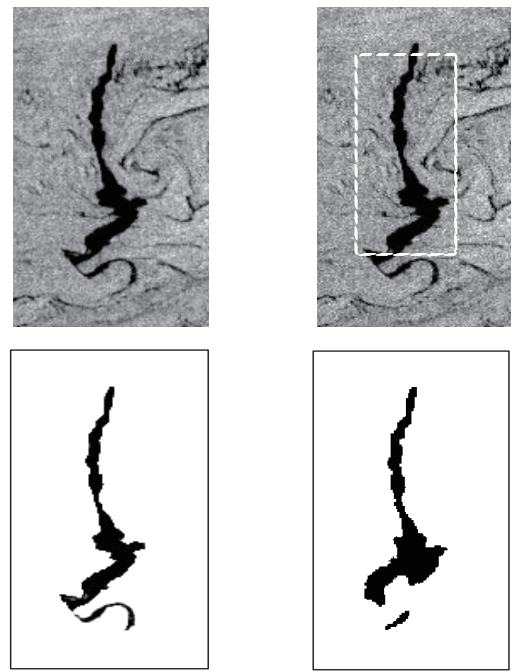

(a)
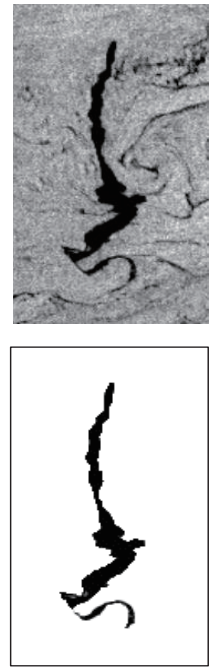

(f)

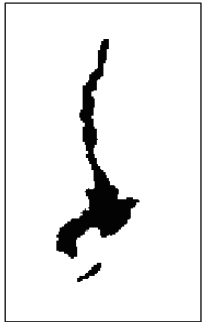

(b)
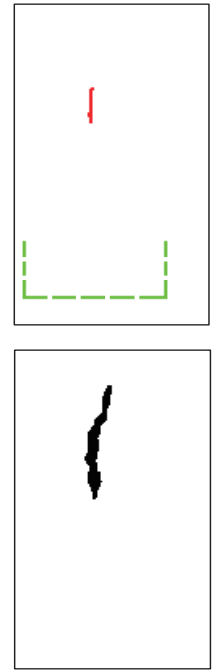

(g)
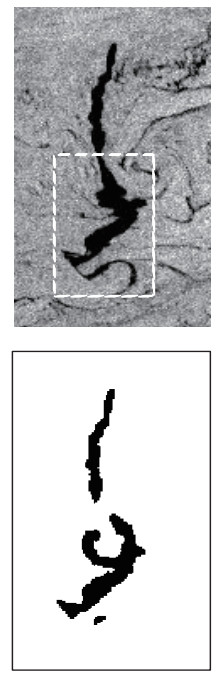

(c)
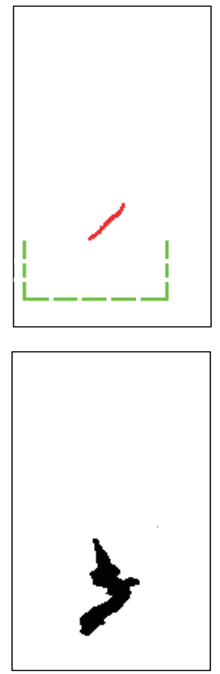

(h)
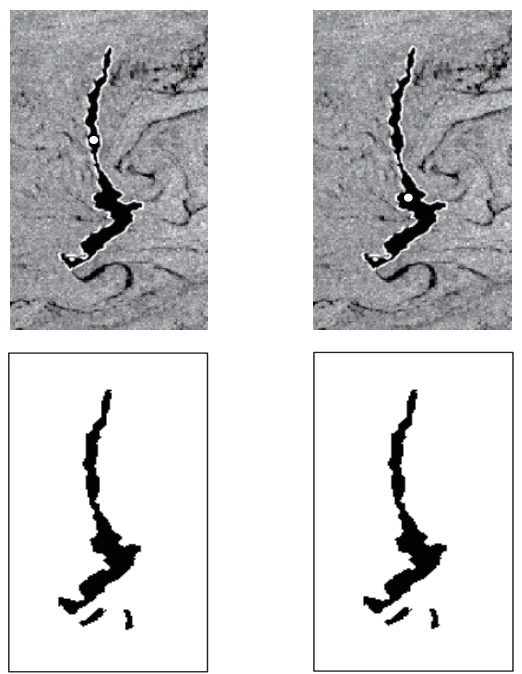

(d)
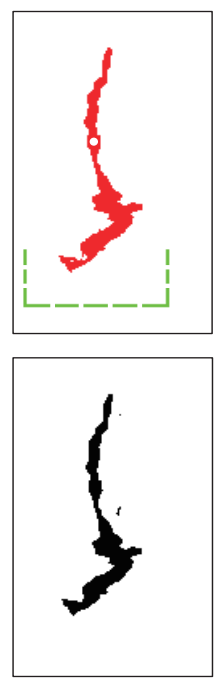

(i)

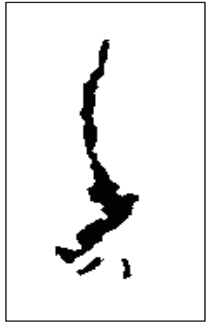

(e)
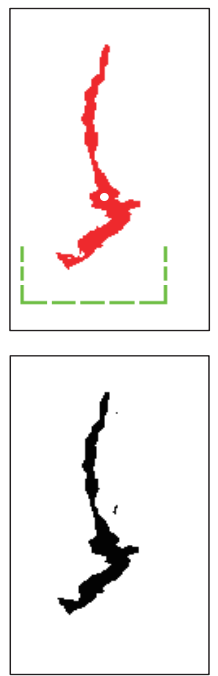

(j)

Fig. 11: Marine oil spill segmentation results using level sets and graph cuts separately. Subfigures are indexed and entitle in the same ways with Fig. 5 .

TABLE I: Marine oil spill segmentation accuracy based on the initialization-free methods.

\begin{tabular}{|c|c|c|c|c|c|c|c|c|}
\hline Accuracy & Image & (a) & (b) & (c) & (d) & (e) & (f) & (g) \\
\hline \multicolumn{9}{|l|}{ Methods } \\
\hline \multirow{2}{*}{ Chan et al. [21] } & Recall & 0.7375 & 0.8404 & 0.9416 & 0.7396 & 0.8000 & 0.9883 & 0.6876 \\
\hline & Precision & 0.3952 & 0.5092 & 0.4096 & 0.9447 & 0.3899 & 0.1533 & 0.5382 \\
\hline \multirow{2}{*}{ Mdakane et al. [16] } & Recall & 0.8297 & 0.8172 & 0.9151 & 0.8345 & 0.8014 & 0.9421 & 0.7886 \\
\hline & Precision & 0.0460 & 0.0513 & 0.0467 & 0.1458 & 0.0227 & 0.1557 & 0.9002 \\
\hline
\end{tabular}


TABLE II: Marine oil spill segmentation accuracy based on level sets.

\begin{tabular}{|c|c|c|c|c|c|c|c|c|c|}
\hline Accuracy & & Image & Fig. 5 & Fig. 6 & Fig. 7 & Fig. 8 & Fig. 9 & Fig. 10 & Fig. 11 \\
\hline \multicolumn{10}{|l|}{ Level sets } \\
\hline \multirow{4}{*}{ Manual initialization } & \multirow{2}{*}{ Recall } & (b) & 0.6517 & 0.7942 & 0.8309 & 0.8506 & 0.4657 & 0.4514 & 0.6208 \\
\hline & & (c) & 0.6651 & 0.3776 & 0.8033 & 0.8195 & 0.4900 & 0.7756 & 0.6077 \\
\hline & \multirow{2}{*}{ Precision } & (b) & 0.3208 & 0.6494 & 0.9667 & 0.9151 & 0.0660 & 0.2139 & 0.6616 \\
\hline & & (c) & 0.3671 & 0.2462 & 0.9907 & 0.9489 & 0.0619 & 0.4028 & 0.7000 \\
\hline \multirow{4}{*}{$\begin{array}{l}\text { One dot fuzzy } \\
\text { initialization }\end{array}$} & \multirow{2}{*}{ Recall } & (d) & 0.8219 & 0.8015 & 0.8736 & 0.7669 & 0.7886 & 0.9377 & 0.8075 \\
\hline & & (e) & 0.8219 & 0.8015 & 0.8736 & 0.7669 & 0.7886 & 0.9377 & 0.8075 \\
\hline & \multirow{2}{*}{ Precision } & (d) & 0.9371 & 0.9229 & 0.9602 & 0.9879 & 0.9452 & 0.9749 & 0.9284 \\
\hline & & (e) & 0.9371 & 0.9229 & 0.9602 & 0.9879 & 0.9452 & 0.9749 & 0.9284 \\
\hline
\end{tabular}

TABLE III: Marine oil spill segmentation accuracy based on graph cuts.

\begin{tabular}{|c|c|c|c|c|c|c|c|c|c|}
\hline Accuracy & & Images & Fig. 5 & Fig. 6 & Fig. 7 & Fig. 8 & Fig. 9 & Fig. 10 & Fig. 11 \\
\hline \multicolumn{10}{|l|}{ Graph cuts } \\
\hline \multirow{4}{*}{ Manual initialization } & \multirow{2}{*}{ Recall } & (g) & 0.1277 & 0.0987 & 0.1750 & 0.0940 & 0.1686 & 0.3077 & 0.2444 \\
\hline & & (h) & 0.1466 & 0.6541 & 0.1383 & 0.1353 & 0.0657 & 0.0496 & 0.4637 \\
\hline & \multirow{2}{*}{ Precision } & (g) & 0.9474 & 0.7642 & 0.9969 & 1.0000 & 1.0000 & 0.9959 & 0.9930 \\
\hline & & (h) & 0.9789 & 0.9179 & 1.0000 & 1.0000 & 1.0000 & 1.0000 & 0.9854 \\
\hline \multirow{4}{*}{$\begin{array}{l}\text { One dot fuzzy } \\
\text { initialization }\end{array}$} & \multirow{2}{*}{ Recall } & (i) & 0.6462 & 0.6151 & 0.8947 & 0.7970 & 0.2686 & 0.6338 & 0.7636 \\
\hline & & (j) & 0.6462 & 0.6151 & 0.8947 & 0.7970 & 0.2686 & 0.6338 & 0.7636 \\
\hline & \multirow{2}{*}{ Precision } & (i) & 0.9681 & 0.9749 & 0.9679 & 0.9930 & 0.9947 & 0.9852 & 0.9612 \\
\hline & & (j) & 0.9681 & 0.9749 & 0.9679 & 0.9930 & 0.9947 & 0.9852 & 0.9612 \\
\hline
\end{tabular}

